\title{
ICT in Medicine and Health Care: Assessing Social, Ethical and Legal Issues
}

\author{
Goran Collste, Penny Duquenoy, Carlisle George, Karin Hedström, \\ Kai Kimppa, Emilio Mordini \\ Linkoping University, Sweden, gorco@cte.org.liu.se \\ Middlesex University, UK, p.duquenoy@mdx.ac.uk \\ Middlesex University, UK, c.george@mdx.ac.uk \\ Örebro University, Sweden, karin.hedstrom@esi.oru.se \\ University of Turku, Finland, kakimppa@it.utu.fi \\ Centre for Science, Society and Citizenship, Italy, e.mordini@bioethics.it
}

\begin{abstract}
Continuous developments in information and communication technologies (ICT) have resulted in an increasing use of these technologies in the practice of medicine and in the provision of medical care. This paper presents a series of perspectives from different areas of expertise on some of the ways in which ICT has changed the social picture in respect of the practice of medicine. The aim of the paper is to provide a context for further debate, in the form of a Panel Session, where the issue of Human Choice and Computing can be discussed with reference to a set of specific scenarios. The authors of this paper represent a wide variety of disciplines including law, ethics, medicine, philosophy and computer science, thus bringing a broad perspective to begin the discussions. The aim of the session is to provoke further discussion, encouraging input from other disciplines respresented by the participants, with a view to identifying the level of human choice in a social arena, which has at its heart a vulnerable community. In this environment, and in this era, the 'social' in social informatics has never been more important.
\end{abstract}

Keywords: social impact, medical informatics, online healthcare.

\section{Overview}

Continuous developments in information and communication technologies (ICT) including the Internet, ambient devices, and intelligent computer systems - have resulted in an increasing use of these technologies in the practice of medicine and in

Please use the following format when citing this chapter:

Collste, G., Dequenoy, P., George, C., Hedström, K., Kimppa, K., Mordini, E., 2006, in IFIP International Federation for Information Processing, Volume 223, Social Informatics: An Information Society for All? In Remembrance of Rob Kling, eds. Berleur, J., Numinen, M. I., Impagliazzo, J., (Boston: Springer), pp. 297-308. 
the provision of medical care. This has led to new concerns regarding the social impact of technology in medicine. Such concerns range from how information technology has changed the practice of medicine and the resulting social consequences, to how the practice of medicine responds to the increasing pervasiveness of technology in our daily lives. The aim of this panel discussion is to identify, review, analyse and debate the social impact of ICT on the practice of medicine. It will focus on various topics such as online medical consultations, online pharmacies, telemedicine, medical information systems, intelligent and ambient medical technologies, and patient autonomy among others. Within all of these topics the central theme of human choice is evident. In some cases technology appears to offer individuals greater choice (for example, online medical consultations, pharmacies, and appliances in the home) and in others the move to technology may constrain individual choice in the practice of health care.

The primary objective of the panel session is to identify the extent to which human choice is encouraged, or diminished, as a consequence of introducing ICT to this specific area. The discussions may reveal that in the health-care field, where the impact of technology can bring both huge benefits and potential disasters, critical choices have to be made. In meeting this objective, and in order to gain a broad perspective, the panel will draw on the expertise of a wide variety of disciplines including law, ethics, medicine, philosophy and computer science. Members will present the current state of affairs from their different perspectives and also comment on possible developments in the future (e.g. electronic implants).

In the following sections the Panel Members describe the areas of their own particular concerns in respect of ICT and the health-care domain, and thus give context to the discussions that all authors hope will form the basis of the session.

\section{Position Statements}

\subsection{Goran Collste}

Professor of Applied Ethics Centre for Applied Ethics, Linköping University,
Sweden

\section{Ethics of e-medicine}

The Internet is more and more used for providing medical information, medical consultation and drug prescriptions. Medical information can be found on an increasing number of medical information site ${ }^{1}$, [Parker \& Muir Gray, 2001; Garpenby \& Hisberg, 2000] patients can consult doctors on line, patients can get access to their medical record through Internet and drugs can be bought on line. Hence, health care is going through a transformation due to different applications of e-medicine.

E-medicine has a number of possible benefits. Patients (and prospective patients) can be better informed about illnesses, drugs and possible treatments. Through consulting an Internet-doctor patients can get a second opinion and become less dependent on local health care. Access to the medical record can give the patients 
quick and accurate information about their health status. These gains are also important from an ethical point of view. For example, one condition for realising the nowadays highly regarded principle of autonomy in health care is that patients are well informed [Beauchamp \& Childress, 1989].

However, it is also possible to envisage a number of ethical problems related to the new development. Internet is a source of medical information but more information is not necessarily beneficial for moral autonomy. Information must be objective as well as comprehended and understood. Do the information sites live up to standards of accuracy and relevance? Pharmaceutical companies own many sites. How does this fact affect objectivity and impartiality? [Silberg, Lundberg \& Musacchio, 1997].

The possibility to consult a doctor on line will have implications for the patientdoctor relationship. The medical encounter has for many years been an issue for discussions in medical ethics [Pellegrino \& Thomasma, 1981]. The relation between doctor and patient is embedded by values of commitment, trust, privacy, confidentiality and responsibility. One can distinguish between different kinds of relationship like, for example, dialogical, instrumental and contractual. In connection to the new possibility of consultation on the Internet one can ask what kind of relation will be established between patient and doctor on line.

A system for an Internet-based patient portal is tried out in Swedish health care. Through the system a patient can get direct access to his/her own medical record. How will this possibility influence the quality of the medical record? Will it perhaps imply that the doctor may leave out some sensitive or harmful information about the patient?

Prescription of drugs is in many countries surrounded by restrictions motivated by solicitude for the patient and avoidance of abuse. Such a policy of weak paternalistic restrictions has a moral basis in principles of non-maleficence and beneficence. The unrestricted marketing of drugs on the Internet runs the risk of undermining this policy.

Common to most kinds of e-medicine is the transcendence of borders. Neither are Internet-based sources of medical information nor Internet-mediated medical consultation restricted to one nation or to one culture. As a consequence the culturally bound values that surround health care are challenged. Hence, the issue of how e-medicine can be ethically assessed must also take the fact of ethical pluralism into consideration.

\subsection{Penny Duquenoy}

Senior Lecturer in Computing and Ethics, Middlesex University, The Burroughs, London, UK

\section{Technology and the self-help patient: issues of competence and understanding}

There is an increasing trend towards utilising the latest developments in technology to facilitate patient self-help and health management. The combination of intelligent systems, hand-held devices and mobile technologies offer a range of applications designed to support patients' independent lifestyles whilst maintaining contact with 
healthcare professionals. Some current examples are: self-monitoring of sugar levels for people with diabetes, together with data link via mobile phone to the medical practitioner [Fleming, 2005]; intelligent monitoring devices in the home for people with potential cognitive difficulties (for example, the elderly) that will alert remote carers [Pollack, 2005]; intelligent materials (used as clothing) that can monitor "vital health data, communicate with remote health centres and present data in a variety of formats for further analysis by doctors and researchers" (EU Project).

These applications are to be applauded in their concept, which is to allow patients the opportunity of living a 'normal' life. In some cases, the diabetes selfmanagement system for example, empowering the patient by showing them patterns of sugar levels, so they can recognise and adapt their habits appropriately. The benefits to the healthcare profession are in allowing monitoring of the patients' condition without the need for physical presence.

However, while these systems help to 'manage' a health situation, they can also reduce patient autonomy. Where technology is employed, and where individuals using this technology are ignorant of how the technology works, it is arguable to what extent they can be said to be 'informed' or in control. The patient is likely to be (in computing science terms) a 'novice user'. That is, a user with little comprehension of how the device works, and its possible consequences. Although there has been, and still is, a great deal of research in the field of usability and computing it is, in most cases, conducted with the 'average' user in mind. We should bear in mind though, that where technology is employed in the medical field, the patient who is the user is likely to be not only a novice, but may have additional difficulties in using technology as a result of either physical or cognitive difficulties.

The patient group represents a particularly vulnerable community, for which competence levels may vary - not only between one individual and another, but also for any one individual over a period of time. For example, the patient may have an illness that affects their cognitive ability at different times (the diabetes patient, for instance). A deficit in cognitive performance may have an adverse effect on their ability to effectively use the technology provided, with possibly disastrous consequences.

A rather less visible concern is where intelligent devices are used in patient care. If these devices are making decisions regarding the health status of the patient (as in the intelligent clothing example), questions relating to decision-making processes must be investigated. If future devices incorporate decision-making methods (such as from Artificial Intelligence, and/or multi-agent systems) it is important to know the basis on which the decisions are being made. Can we be sure, for example, that the data, which informs the decision, is accurate? Some thought should also be given to what constitutes a 'decision' - this is particularly relevant in terms of patient personal data, whereby the Data Subject has "The right to prevent decision making solely by automatic means" (UK Data Protection Act).

Incorporating complex technological systems into the healthcare picture, and in particular placing these systems in the hands of the patients, creates a tension. On the one hand the systems are aimed at benefiting the patient, and on the other they place an extra burden on the patient in terms of technological understanding and management. 
From the point of view of the health-care professional, how are they to ascertain 'informed consent' if the patient does not understand how the technology works, or its possible consequences? Will the technology have to be explained to patients, together with all the implications of data transfer and medical impact (in the physiological sense). What are the criteria for informed consent? Should a list be devised? How will it be judged that such consent has been given (and that the patient fully understands the information that has been given)?

Whilst these technologies may be welcomed by practitioners and patients alike, offering increased levels of independence for some health conditions, that independence carries further responsibilities. The patient needs to understand not only the operation of the technology with regard to their own condition, but also in relation to the wider world. Modern devices can have an impact on other devices, as the following 'safety information' [Nokia, 2005] makes clear:

"Operation of any radio transmitting equipment, including wireless phones, may interfere with the functionality of inadequately protected medical devices. Consult a physician or the manufacturer of the medical device to determine if they are adequately shielded from external RF energy or if you have any questions. Switch off your device in health care facilities ...

Pacemaker manufacturers recommend that a minimum separation of $15.3 \mathrm{~cm}$ be maintained between a wireless phone and a pacemaker to avoid potential interference ... If you have any reason to suspect that interference is taking place, switch off your device immediately.

Hearing aids: some digital wireless devices may interfere with some hearing aids. If interference occurs, consult your service provider ..."

[Source: instruction booklet provided with Nokia 6680, November 2005.]

Note how it is the user who has to (a) understand and know that their device (in this case an ordinary mobile phone) may cause problems, and (b) take steps to deal with it (consult their physician, manufacturer, or service provider, or switch it off). If hand-held devices are to be used for self-management of health care the problems of radio transmission outlined above could well apply - not just to the patient, but also to others in their vicinity. In the case of the mobile phone extract quoted above the responsibility is on the user, and it should be remembered that our user - the patient - is by definition unwell. They may find it difficult to cope, both with the information received and with managing their devices, thus increasing stress. Further work is needed to assess the capability of patients (and healthcare professionals) in using the new technologies, to determine appropriate levels of training, and above all to recognise that technology may not be the best answer for all patients.

\subsection{Carlisle George}

Barrister and Senior Lecturer in Computing and IT Regulation, Middlesex University, The Burroughs, London 


\section{ICT in healthcare: some legal concerns}

Information and communication (ICT) technologies in medicine and medical care arguably bring benefits to medical practitioners and patients [e.g. see Hodge et al, 1999]. There are, however, many legal concerns about the use and operation of these technologies which include the Internet (e.g. online pharmacies, telemedicine, email) and information systems (e.g. computerised databases holding electronic patient records and other medical data). This discussion will focus on some legal issues related to use of these two technologies.

The Internet is increasingly being used to provide healthcare, in various ways including the operation of Internet Pharmacies. An important legal concern with Internet Pharmacies is whether medical practitioners and pharmacists providing online/distant services are licensed to practice their respective professions in the respective jurisdictions where they and their patients are located. Related to this is issue is whether drugs offered to patients are legally approved in the jurisdiction where the patient is located. Internet Pharmacies also provide online consultations (via online questionnaires), which are used as a basis for issuing prescriptions and selling prescription drugs. The writing of prescriptions via online consultation raises important legal issues especially related to confidentiality and civil liability for medical malpractice should something go wrong [Kahn et al, 2000]. With regard to confidentiality, information given for online consultations may be prone to be seen by people other then the consulting doctor, unless strict security and protocols are in place. Patients may have no way of knowing whether or not such breaches have occurred and hence may not be able to address them. With regard to civil liability, it may be difficult to clearly establish malpractice where an online prescription is issued. This is because whereas in a traditional doctor-patient relationship a clear duty of care exists, it is debatable whether a doctor who prescribes medication online (without any direct verbal or physical contact with a patient), forms a doctor-patient relationship and therefore attracts a duty of care [Kahn et al, 2000]. In view of the above, can online patients, legally address issues of medical malpractice, especially where the medical practitioner is located in a distant nation state? If not, what legal mechanisms need to be put in place to address this issue?

Telemedicine involves the use of ICT to deliver health care (information and services) to patients separated (from medical providers) by geographic boundaries [Bashshur, 1995]. A main legal concern with telemedicine is the issue of jurisdiction in terms of (a) whether a medical provider has the necessary license to practice in the jurisdiction where he/she is situated and also where the patient is situated; (b) determining the procedural issue of where an action can be brought against a medical provider for malpractice. Within the EU jurisdiction is addressed by the Brussels Regulation, which determines where actions in tort and contact can commence, however, this may be more difficult to resolve where countries not governed by the Brussels Regulation are involved. Another legal concern is the possible liability for malpractice for either transmitting or receiving an inaccurate telemedical opinion [Hodge et al, 1999]. This raises issues such as: the timing and nature of the doctorpatient relationship; and the reliability of technology used in telemedicine. How can one legally determine when a doctor-patient relationship begins and the extent of the duty of care owed to the patient? How can one legally address errors which may be 
due to either (or a combination of) the doctor's medical competence, the failure to use the technology properly or the failure/reliability of the technology itself? One electronic technology used in telemedicine is email. Use of email in telemedicine raises the legal issue of doctor-patient confidentiality among other issues [see Spielberg, 1998] since a doctor has a duty to keep all patient information confident. Emails are subject to being intercepted during transmission and can be read by others having access to the doctor's mailbox. Such privacy breaches are usually very difficult to prosecute due to the need for adequate evidence (such as security trails). The contents of emails can also form part of a patient's medical record and emails are recoverable from servers even when deleted. Are patients adequately informed of the potential issues regarding email communication? Who is responsible for compensating the patient if email correspondence is compromised during transmission?

Information systems in healthcare are often used to store, access and transmit electronic medical data. These activities include implementing computerised databases and facilitating data exchange. Compared to physical records, electronic records can be easily accessed (by many people in different locations), searched, changed, copied and transmitted across networks. Also, inadequate security can result in unauthorised access and interception of communications (especially email). The above raises many legal concerns such as maintaining the: privacy of patient (identifiable) data; quality and reliability of patient data; and confidentiality (obligation in both tort and contract) of patient information [Hodge et al, 1999].

The protection of medical data is extremely important since unauthorised access, modification or disclosure can adversely affect a patient (e.g. wrong treatment, stigmatisation, discrimination). In the European Union (EU), personal data (collected from a 'data subject') is protected under data protection legislation, which address issues such as subjects' rights regarding disclosure of personal data, limitations on disclosure without consent, duty to maintain accuracy and integrity of personal data, the provision of adequate security against unauthorised access of personal data, and strict conditions for the transfer of personal data outside the EU. The effectiveness of EU data protection law, however, must be examined in light of the many exceptions which are given in the Act. For example under the United Kingdom (UK) Data Protection Act 1998 (which implements EU legislation), 'sensitive personal data' (which includes medical records, racial origin, criminal records among others) must not be processed without the explicit consent of data subject. The law, however, makes an exemption to this requirement where the processing of data is necessary for medical purposes and is carried out by a health professional or anyone owing a duty of confidentiality (Data Protection (Processing of Sensitive Personal Data) Order 2000, Article 8(5)). This exemption clearly introduces a weakening of subjects' rights since the 'processing' of data includes a variety of activities such as amending, augmenting, deleting, re-arranging and extracting information (Data Protection Act 1998, Section 1(7)). While this is counterbalanced by the lawful provision of subject access to medical records in the $1998 \mathrm{Act}$, the law also exempts the grant of access, if in the opinion of a relevant health professional such access would result in serious physical or mental harm to the data subject or any other person (The Data Protection (Subject Access Modification) (Health) Order 2000). Does UK/EU data protection legislation provide the right balance between patients' 
rights and the needs of the medical profession? Should patients have an absolute legal right to their records?

\subsection{Karin Hedström}

Senior Lecturer, Department of Informatics, Orebro University, Sweden.

\section{IT as a prerequisite for the realization of care services}

Earlier research of IT in elderly care has often viewed IT as a tool for administration and management [e.g. Beck, 1997], and not as a way of providing support that facilitates the care worker's meeting with the elderly. This is in line with the prevalent view of administrative tasks in elderly care, where it is common, notably in organization wide IT systems, to separate administrative tasks and care activates. Administration and the use of IT systems for coordination and administration are frequently viewed as an obstacle that prevents care workers from doing their 'real job'. Administration is not always well integrated in the care related activities and thus not seen as part of the care work. Administrative tasks as well as its tools are often treated and viewed as separate from the core of care work. Administration is seen as a negative activity that takes time from the 'real work'.

IT systems and administration are often seen as separate from the care giving organisation, and care professionals are furthermore often forced to prioritize administrative tasks on the expense of more care related tasks, with antagonism against the new IT system as a consequence [see also Wilson, 2002]. Care tasks and administrative tasks are often inadequately integrated, both in relation to the care worker's professional role and the content of care work.

To use the concept 'administration', instead of for instance 'information transfer' or 'communication', which describes the functions of the IT systems in elderly care more accurate, indicates alienation and distancing. To 'administer' is often, by the care workers, viewed as a destructive activity, even though it mostly involves necessary tasks such as to be informed and inform others. Care work requires sufficient and accurate information, which makes administration, in the form of communication and knowledge transfer, vital. To communicate, orally or via documents or tools such as IT systems, is necessary in order to provide safe and high quality care. Why is administration seen as such a negative work task? And why are there still so many 'failed' IT systems in healthcare? Another interesting question is the role of the care professional in relation to IT? What is it to produce care services? What is included and what is the role of the care professional?

\subsection{Kai Kimppa and Janne Lathtiranta}

Kai Kimppa: Lecturer, Department of Information Technology, University of Turku and Turku Centre for Computer Science (TUCS/LABORIS) focusing on the ethical aspects of ISs. 


\section{Health and Medical Informatics and Responsibility: What are the Roles of Anthropomorphism and Informed Consent in a Distributed World?}

Anthropomorphism, or humanisation, of the artefacts of ICT used in medicine and health care, has an impact on the relationship between the patient and the health care professional [Lahtiranta \& Kimppa, 2004]. Anthropomorphism can manifest in many different ways: the artefacts can be designed to interact with the user in a human-like manner, or the artefacts can encapsulate the decision making process behind a result or recommendation, creating an illusion that the information is provided by a human expert and not by, for example, some medical instrument or application. In addition, the artefacts are often anthropomorphised by the users themselves, it is not uncommon to hear a user to blame a system or application by saying 'it is not my fault - the machine did it!'

The decisions originating from an artefact of ICT used in medicine or in health care should be analyzed by a trained professional(s) and in practice never relied upon automatically. This is usually the case, but there are situations where it is nearly impossible for the verification process to be executed by a human. For example, in calculation-intensive work the verification is sometimes beyond human capabilities. The artefact could also be implemented in such fashion that the professional is intentionally removed from the decision making process, such as with certain 'blackbox applications' used in diabetes. These applications analyze patient's condition, calculate and ration the medication to be applied by the patient automatically.

The fact that the artefacts of ICT used in health care or medicine are used to overcome geographical distances and cultural barriers creates a problematic situation. First, this brings forth an issue of who (or even what) answers to the consultation request. Second, it raises the question of relevant authority; the answer may originate from a location which belongs to a completely different jurisdiction from the one the request was submitted. Should a problem arise with the given treatment, which legislation is to be used? Third, reliance to the given advice, or overriding advice of a health care professional with it, could lead to problems. If the professional relies to the advice and the treatment is not successful, this can be pointed out and the professional could be accused of over-relying on the advice. On the other hand, should the health care professional choose to override the advice because of their professional expertise, the system typically can be shown to be consulted, and overwritten, and a malpractice suit be brought forth. These kinds of reliability problems are not just a matter of possibly near-future expert systems based on artificial intelligence technologies, but more closely to the everyday hospital information systems, if the source of the advice is not known or it is obscure by nature.

If the health care professional cannot always be aware of the source of information, consultation or recommendation, how could the patient? With who (or what) does the patient-health care professional form a relationship? Surely not with an artefact of ICT. With the party responsible of implementing the original health care procedures on the artefact? With the party who decided that this kind of artefact could be utilized in this particular situation? What kind of (informed) consent issues should be taken into account? These questions are more current now than ever before due to the ongoing health care paradigm shift in the western highly industrialized 
countries where responsibility of one's personal care is becoming more and more one's personal matter.

\subsection{Emilio Mordini}

\section{Medical doctor and Coordinator of the EU funded project BITE-Biometric Identification Technology Ethics (www. biteproject.org).}

\section{Automatic Identification Technology in Medical and Social Care}

Accurate identification and verification of identity is important at many levels in social and medical care. First the need to administrate scarce resources in social and medical care creates an imperative to avoid the illicit use of social welfare and medical support. Within a few decades, nearly half the European population will be at retirement age or beyond, placing a great burden on the European economy. Identification technologies will increasingly play a critical role as a gatekeeper of future healthcare and social services. Departments in charge of social assistance in countries like Spain and the Netherlands are already launching programmes for detecting and preventing duplicate benefits. This is a kind of fraud that involves the collection of more benefits than one is entitled to, by entering the program under two or more identities. A wide consensus appears to exist concerning the high levels of this type of fraud, and heighten the urgency for establishing new identification practices. It is claimed that the introduction of identification / verification of identity technologies would result in billions of savings on public spending. Unauthorized use of assistance programmes (e.g., heroin addicts who participate in methadone maintenance plans) could be tackled by using automatic systems for identification (both to authenticate people and to track medications, for instance by using RFID or other electronic tags). In addition, people are accessing more and more social services over the Web; for this to be secure, establishing people's identity is essential. Finally, among the most important healthcare issues that directly affect patient safety and quality of care are the ability to correctly identify patients and to confirm the accurate delivery of clinical services for those patients. In health care we need to know that the clinician is administering the right medication to the right patient, each and every time. Identification technologies such as biometrics and RFID are already in use to identify and track special categories of patients in hospitals. Pilots are in progress in Italy, Spain and the Netherlands. RFID has been in use since 2004 in California to track organs for transplants. Concerns have been raised about the ethical and social implications of identification technologies, when they touch areas of personal life where one would not ordinarily expect to be identified and tracked, such as healthcare and social services. In this area, individuals must face a decision to trade their personal data for gain, affecting the balance between ease of use and levels of security and protection of privacy. Citizens often must waive their data protection rights if they wish to receive many services. This is not purely a privacy issue, because it involves important ethical questions, such as the conflict between the individual's autonomy and social pressure. On the other hand, there is also a conflict between personal freedom to trade one's personal data and what society at large considers desirable or ethically acceptable in this field. 


\section{Conclusion}

The position statements above have focused on various perspectives on ICT in medicine and health care. They are a critical reflection on aspects of the politics of the information society, because they discuss important issues regarding the effect of information technology use on the individual and society as a whole. They also focus on the common theme of 'human choice' whether viewed from an ethical, legal or social dimension. It is hoped that the contributions will provide a springboard for interesting discussions which will bear fruitful solutions to address some of the concerns raised.

\section{References}

Bashshur, L (1995) On the definition and evaluation of telemedicine. Telemed $J$ 1995; 1:1930.

Beauchamp, T L \& Childress, J F, (1989). Principles of Biomedical Ethics, Oxford: Oxford University Press

Beck, E. (1997). Managing Diffracted Rationalities: IT in a Home Assistance Service. Technology and Democracy: Gender, Technology and Politics in Transition? Moser, I. and Aas, G.H., Eds, Centre for Technology and Culture, University of Oslo, Oslo: 109132.

EU Project: WEALTHY, Source: Virtual Medical Worlds, Monthly Newsletter. http://www.hoise.com/vmw/05/articles/vmw/LV-VM-06-05-37.html. (06/12/2005)

Fleming, Nic. 'Mobile phone link reduces chance of diabetes problems' 29/08/2005 at http://www.telegraph.co.uk/news. (06/12/2005)

Garpenby, P \& Hisberg, M, 2000, Hälsoinformation idag och imorgon, CMT Rapport 2000:3, Linköpings universitet, Linköping (unpublished)

Hodge, J, Gostin, L and Jacobson, P (1999). Legal issues concerning electronic Health information: Privacy, Quality and Liability. JAMA October 20, 1999, Vol 282, No 15.

Kahan, S, Seftel A and Resnick, M (2000) Sildenafil and the Internet. The Journal of Urology, Vol. 163, 919-923, March 2000

Lahtiranta, J. \& Kimppa, K. (2004) Telemedicine and responsibility: why anthropomorphism and consent issues muddle the picture. The 5th International We-B (Working For EBusiness) conference, 25-26 November, Perth, Western Australia.

Parker, M \& Muir Gray, J A (2001). What is the role of clinical ethics support in the era of emedicine?, Journal of Medical Ethics, 2001; 27, suppl 1:i 33-35

Pellegrino, E D \& Thomasma, D C, 1981 A Philosophical Basis of Medical Practice, Oxford, Oxford University Press

Pollack, Martha E. (2005) 'Intelligent Technology for an Aging Population' AI Magazine, Vol.26:2, Summer 2005.

Silberg, W M, Lundberg, G D, Musacchio, R A (1997) Assessing, Controlling, and Assuring the Quality of Medical Information on the Internet, Journal of American Medical Association, 277:1244-1245

Spielberg, A (1998) On Call and Online, JAMA October 21, 1998, Vol 280, No 15.

Wilson, M. (2002). 'Making nursing visible? Gender, technology and the care plan as script.' Information Technology \& People 15(2): 139-158. 
308 Goran Collste, Penny Duquenoy, Carlisle George, Karin Hedström, Kai Kimppa, Emilio Mordini

Notes

1 There are currently between 15,000 and 100,000 health-related sites in Great Britain and they have been visited by approximately 30 million people. A Swedish survey showed that of those that accessed the Internet, about $20 \%$ had been looking for health-related information (see Parker \& Muir Gray, 2001; Garpenby \& Hisberg, 2000). 